Vol. 50 (1994) [197-204]

\title{
RINGS WITH QUASI-INJECTIVE IDEALS
}

\author{
George Ivanov
}

\begin{abstract}
A short proof determining the structure of $Q$-rings with infinite identities is given. The structure of $p Q$-rings is determined and it is shown that essentially all $w Q$ rings with finite identities are $p Q$-rings.
\end{abstract}

Rings with an abundance of quasi-injective modules have been studied extensively since the late 1960's. Of particular interest are those rings whose (left) ideals are quasiinjective, the (left) $Q$-rings. It follows from the author's work in [3] that the study of $Q$-rings reduces to the study of the following 3 disjoint classes.

I. local $Q$-rings;

II. indecomposable $Q$-rings with more than one idempotent; and

III. $Q$-rings with an infinite set of orthogonal idempotents.

A ring's identity is finite if it is a sum of primitive orthogonal idempotents, otherwise it is infinite. It was further shown that the rings of class II have finite identities and are only the simple Artinian rings and the rings

$$
H(m, D, V)=\left(\begin{array}{ccccc}
D & & & & V \\
V & D & & & \\
& V & \ddots & & \\
& & \ddots & D & \\
& & & V & D
\end{array}\right) \quad \text { with } m \text { rows, for } m \geqslant 2
$$

where $D$ is a skew field and $V$ is a null $D$-algebra, one dimensional on both sides. The rings of class $I$ are the local left self-injective left duo rings.

The structure of $Q$-rings of class III is determined in Section 1. It follows from that result that the conjecture in [4] is false. This structure theorem was obtained independently by Byrd [1] using different methods. The author's proof (which was obtained in 1976) appears to pre-date Byrd's and is very much shorter and simpler.

Received 12th October, 1994

Honorary Associnte at Macquarie University. The present note is a revised version of an unpublished article written at Sydney University in 1976.

Copyright Clearance Centre, Inc. Serial-fee code: 0004-9729/94 \$A2.00+0.00. 
It is included in this note, as its methods may be of use in studying other rings with infinite identities - usually an intractable task.

In Section 2 two generalisations of $Q$-rings are studied: $p Q$-rings - rings whose proper left ideals are quasi-injective; and $w Q$-rings - rings whose left ideals not isomorphic to the ring itself are quasi-injective. These were first studied in [5] and [6], respectively. It is shown that the only non-local $p Q$-rings which are not $Q$-rings are the rings $\left(\begin{array}{ll}D & 0 \\ D & D\end{array}\right)$ where $\mathrm{D}$ is a skew field. It is also shown that the only $w Q$-rings with finite identities which are not $p Q$-rings are those with a unique idempotent (the identity).

Throughout this note all rings have identity and all ideals and modules are left and unital. The letters $e, f$, with or without subscripts or superscripts, always denote idempotents and $E(M)$ denotes the injective hull of a module $M$. The methods used were developed in [3] so most of the arguments will be given only in outline.

\section{Q-RINGS OF Class III}

Throughout this section $R$ is a $Q$-ring of class III. The crucial result is the following generalisation of Lemma 3 of [3]. It does not require the assumption that $R$ is of class III. This is essentially Theorem 5 of [1] which is the primary contribution of that paper. Its proof is both long and difficult.

Lemma 1.1. Let $\left\{M_{i} \mid i \in I\right\}$ be a set of minimal ideals of $R$ with injective hulls (in $R$ ) $R e_{i}, i \in I$, respectively. For only finitely many $i$ is $M_{i}$ an image of $\dot{R}\left(1-e_{i}\right)$.

Proof: Assume the converse, that $I$ is infinite and that each $M_{i}$ is an image of $R\left(1-e_{i}\right)$. By Lemma 3 of [3] only finitely many $M_{i}$ are images of the $R e_{j}$ so it may be assumed that none are. The $M_{i}$ are mutually non-isomorphic since if two are isomorphic, say $M_{k}$ and $M_{l}$, then by Lemma 2 of [3] they would be injective and hence images of $R e_{k}\left(=M_{k}\right)$, a contradiction to the last assumption. Hence if $K_{i}$ is the kernel of an epimorphism $R \rightarrow M_{i}$ then the $K_{i}$ are distinct maximal ideals. For an arbitrary pair $i, j \in I$ there is an idempotent $f_{j} \in K_{i}$ with the property that $f_{j} M_{j} \neq 0$ and $\left(1-f_{j}\right) M_{i} \neq 0$. One of $f_{j},\left(1-f_{j}\right)$ satisfies the equation $x M_{k} \neq 0$ for infinitely many $k$. Denote this idempotent by $f_{1}^{\prime}$ and the other by $f_{1}^{\prime \prime}$. If $f_{1}^{\prime \prime}=f_{j}$ then there is an idempotent $f_{1} \in R f_{j} \cap R\left(1-e_{j}\right)$ with the property that $f_{1} M_{j} \neq 0$. If $f_{1}^{\prime \prime}=1-f_{j}$ then there is an idempotent $f_{1} \in R f_{1}^{\prime \prime} \cap R\left(1-e_{i}\right)$ with the property that $f_{1} M_{i} \neq 0$. Note that in either case $f_{1}$ and $f_{1}^{\prime}$ are orthogonal and $R f_{1}$ has a non-zero image in $R\left(1-f_{1}\right)$. Repeat the above procedure with $M_{i}$ and $M_{j}$ replaced by ideals $M_{l}$ which are images of $R f_{1}$ to obtain orthogonal idempotents $f_{2}, f_{2}^{\prime} \in R f_{1}$ with the properties that $R f_{2}$ has a non-zero image in $R\left(1-f_{2}\right)$ and $f_{2}^{\prime} M_{l} \neq 0$ for infinitely many $l$. In this fashion one can construct an infinite set $\left\{f_{n}\right\}$ of orthogonal idempotents with the 
property that each $R f_{n}$ has a non-zero image in $R\left(1-f_{n}\right)$. This contradicts Lemma 3 of [3] and proves the lemma.

LEMMA 1.2. Let $\left\{e_{i}\right\}$ be an infinite set of orthogonal central idempotents of $R$ and let $R e$ be an injective hull in $R$ of $\oplus R e_{i}$. Then $R e=\prod e_{i} R e_{i}$.

Proof: As each $R e_{i}$ is injective $\prod R e_{i}$ is injective and as each $e_{i}$ is central, $\oplus R e_{i}$ is an essential submodule of $\prod R e_{i}$. Therefore $R e$ is isomorphic to $\prod R e_{i}$. Since $(1-e) R e=0, R e=e R e \cong \operatorname{End}(R e) \cong \operatorname{End}\left(\prod R e_{i}\right)$. As the $e_{i}$ are orthogonal and central $\operatorname{End}\left(\prod R e_{i}\right) \cong \prod e_{i} R e_{i}$ and $e_{i} R e_{i}=R e_{i}$, thus $R e=e R e=\prod e_{i} R e_{i}$ as required.

To simplify the statement of the main result we need the following definition.

Definition: Let $C$ be a $Q$-ring with infinite identity and all of whose idempotents are central, $U$ a simple left $C$-module, $D$ the endomorphism ring of $U$, acting on the right, so that $U$ is a $(C, D)$-bimodule, and let $V$ be a null $D$-algebra, one dimensional on both sides. Then we define $H(m, C, U, D, V)$, for an integer $m \geqslant 3$, to be the following matrix ring.

$$
H(m, C, U, D, V)=\left(\begin{array}{lllll}
D & & & & \\
V & \ddots & & & \\
& \ddots & D & & \\
& & V & D & \\
& & & U & C
\end{array}\right) \quad \text { with } m \text { rows and columns. }
$$

For $m=2$ we define $H(m, C, U, D, V)$ to be the ring $H(2, C, U, D)=\left(\begin{array}{ll}D & 0 \\ U & C\end{array}\right)$. For $m=1, H(m, C, U, D, V)$ is simply the ring $C$.

We can now state and prove the main theorem.

THEOREM 1.3. A ring $R$ is a $Q$-ring of class III with no summands of class II if, and only if, it is isomorphic to a product of a finite number of rings $B(m, C, U, D, V)$ each of which satisfies the following conditions:

(i) $e U=0$ for every primitive idempotent $e \in C$,

(ii) $C=C_{0} \times C_{1}$ where $C_{0}$ is an infinite or empty product of local rings and $C_{1}$ has no primitive idempotents.

Proof: Let $\mathcal{M}=\left\{M_{i} \mid i \in I\right\}$ be the set of minimal ideals with the property that $\left(1-e_{i}\right) M_{i} \neq 0$, where $R e_{i}$ is the injective hull in $R$ of $M_{i}$. By Lemma 1.1 the set $I$ is finite and so $R f$ is the injective hull in $R$ of $\underset{i \in I}{\oplus} M_{i}$, where $f=\sum_{I} e_{i}$. Hence by Lemma 2 of [3], $f R(1-f)=0$ and all idempotents in $(1-f) R(1-f)$ are central in $(1-f) R(1-f)$. 
Let $\left\{f_{j} \mid j \in J\right\}$ be the set of primitive idempotents in $C=(1-f) R(1-f)$. Being central, these idempotents are mutually orthogonal. If $R e$ is the injective hull of $\oplus R f_{i}$ then, by Lemma 1.2, $R e=\prod R f_{i}=\prod f_{i} R f_{i}$. It follows that $C=C_{0} \times C_{1}$ where $C_{0}=\prod f_{i} R f_{i}$ and $C_{1}=R(1-f-e)=(1-f-e) R(1-f-e)$ has no primitive idempotents.

Let $\mathcal{M}_{0}=\left\{M_{i} \mid i \in J\right\}$ be the subset of $\mathcal{M}$ consisting of those minimal ideals which are images of $C$. We now show that $C=\oplus C_{i}$ where the $C_{i}$ are subrings with the property that $C_{i} M_{i}=M_{i}$ and $C_{j} M_{i}=0$, for $i \neq j$. If $F=C / J(C)$ then since each $M_{i}$ is annihilated by a maximal, ideal each $M_{i}$ is canonically an $F$-module. Let $K_{i}$ be the annihilator of $M_{i}$ in $F$ and let $a \in K_{2} \backslash K_{1}$. Then $a M_{1}=M_{1}$ and $a M_{2}=0$. Since $F$ is a regular ring (Theorem 5.1 of [2]) there is an idempotent $e \in F$ such that $F e=F a$. Therefore $M_{1}$ is an image of $F e$ and $M_{2}$ is an image of $F(1-e)$. But $e$ is central in $F$ so $F$ is a direct sum of the subrings $F e$ and $F(1-e)$. In this way we can obtain a decomposition $F=\oplus F_{i}$ where the $F_{i}$ are subrings with the property that $F_{i} M_{i}=M_{i}$ and $F_{i} M_{j}=0, i \neq j$. As idempotents lift modulo $J(C)$ (Theorem 5.6 of [2]) the required decomposition of $C$ follows.

Let $M_{j 0} \in \mathcal{M}_{0}$ be the image of $C_{j}$ and let $M_{j 1}$ be the image of $R e_{j 0}=E\left(M_{j 0}\right)$; let $M_{j 2}$ be the image of $R_{j 1}=E\left(M_{j 1}\right)$ and so on. This sequence is finite. As $\mathcal{M}_{0}$ is finite it is sufficient to show that each $M_{j i}$ can appear only once. If not then one of these minimal ideals is an image of two $R e_{j i}$ or of $C_{j}$ and an $R e_{j i}$. In both cases $R$ would have two isomorphic indecomposable injectives and thus would contain a simple Artinian ring as a summand (Lemma 2 and Theorem 1 of [3]) - a contradiction to the assumption that $R$ has no summands of class II. If $R f_{j}=C_{j}+R e_{j 0}+R e_{j 1}+\ldots$ then $R f_{j}$ is a two sided ideal and a ring summand of $R$. For by construction $R f_{j}$ has no images in $R\left(1-f_{j}\right)$ and $R\left(1-f_{j}\right)$ can have no images in the $R e_{j i}$, by the above argument, and no images in any $C_{i}, i \neq j$, since all idempotents in $C$ are central. The minimal ideals in $\mathcal{M}$ must be exhausted by the $R f_{j}^{\prime} s$ as otherwise $R$ would have a summand of class II. Hence $R$ is a direct product of the rings $R f_{j}=R_{j}$. From now on we only consider these rings.

The matrix representation of $R_{j}$ is obtained, in the usual manner, by considering it as its own ring of endomorphisms. The proof that the injective hull of each $M_{j i}$ has a unique submodule (namely $M_{j i}$ ) is the same as the second paragraph of the proof of Lemma 4 of [3]. The rest of the details of the matrix representation of $R_{j}$ are similar to the proof of Theorem 3 of [3]. We now show that condition (i) is satisfied.

Assume it is not. Then there is a primitive idempotent $e \in C$ such that some $M_{i}$, say $M_{1}$, is an image of $R e$. Let $M_{2}$ be an $M_{i}$ which is an image of $R e_{1}=E\left(M_{1}\right), M_{3}$ an $M_{i}$ which is an image of $R e_{3}=E\left(M_{2}\right), \ldots$ and so on. As the number of the $M_{i}$ is finite this process terminates at step $n$, say. That is, $R e_{n}$ has no images outside itself. 
Therefore $B=R e \oplus R e_{1} \oplus \cdots \oplus R e_{n}$ is a two-sided ideal of $R$. But $B$ has at least two idempotents and is indecomposable so it is a $Q$-ring of class II: a contradiction to the hypothesis that $R$ has no summands of class II.

The proof of the converse is similar to that for Theorem 3 of [3].

COROLlary 1.4. A left $Q$-ring need not be right injective.

Proof: The right ideal $\left(\begin{array}{l}D \\ \end{array}\right)$ in the ring $\left(\begin{array}{ll}D & \\ U & C\end{array}\right)$ is not injective.

The following Corollary is the correct statement of the Remark at the end of [3].

Corollary 1.5. A ring is a $Q$-ring if, and only if, it is a sum of (a finite number) of rings of class $I$ and rings of type $H(m, C, U, D, V)$ which satisfy the conditions of Theorem 1.3.

Proof: By Lemma 3 of [3] a $Q$-ring can have only a finite number of central idempotents each of which generates a ring of class II. Hence a $Q$-ring is a finite sum of rings of class II and the rings $H(m, C, U, D, V)$.

Note that products of rings of class I are contained in the rings $H(m, C, U, D, V)$ as part of the subrings $C$.

COROLlary 1.6. A ring is a left and right $Q$-ring if, and only if, it is the sum of rings of class $I$ and a ring $Q=Q_{1} \times Q_{2}$ all of whose idempotents are central and which has the following properties. $Q_{1}$ is a product of local left and right $Q$-rings and $Q_{2}$ is a left and right $Q$-ring with no primitive idempotents.

Proof: The rings of class II are left and right $Q$-rings and the rings $H(m, C, U, D, V)$ are not right injective if $m \geqslant 2$. So the only rings of the latter type that can appear are the rings $C$.

\section{2. $p Q$-RINGS AND $w Q$-RINGS}

The study of $p Q$-rings was initiated in [5]. The authors showed that prime $p Q$ rings are $Q$-rings and that commutative Noetherian $p Q$-rings are $Q$-rings except for a, somewhat trivial, case. It was shown in [6] that nonsingular $w Q$-rings satisfying certain finiteness conditions are either $Q$-rings or the matrix rings in Theorem 2.3. Those finiteness conditions imply that the rings have finite identities. In this section we determine the structures of arbitrary $p Q$-rings and of $w Q$-rings with finite identities.

There are trivial examples of $p Q$-rings and $w Q$-rings with unique idempotents which are not $Q$-rings. The available techniques seem to be unable to shed much light on the structure of these rings or, in fact, on local $Q$-rings. So the major remaining open questions are to determine the structures of these rings and of $w Q$-rings with infinite identities. Some progress is made on the last question in [7] where the authors 
obtain some results on arbitrary nonsingular $w Q$-rings, but even their structure remains unknown.

We first study $p Q$-rings and show that, apart from some simple exceptions, they are $Q$-rings.

Lemma 2.1. If $R$ is a $p Q$-ring with a unique idempotent (the identity) then $R$ is a local ring and every element in its radical is a zero divisor.

ProOf: Let $a \in R$ be an element which is not a zero divisor. Then $R a \cong R$ so if $R a \neq R$ then $R a$, and hence $R$, is quasi-injective. This implies that $R$ is a local ring (Proposition 5.8 of [2]) and every element in its radical is a zero divisor (Theorem 5.1 of [2]): a contradiction. Hence every regular element of $R$ is a unit. Therefore, $R$ is a local ring and its radical consists of zero divisors.

Lemma 2.2. If $R$ is a $p Q$-ring with at least three orthogonal idempotents then it is a $Q$-ring.

Proof: Let $e_{1}, e_{2}, e_{3}$ be three orthogonal idempotents whose sum is the identity of $R$ and let $L$ be a left ideal of $R$. To show that $R$ is self-injective it is sufficient to show that any homomorphism $\phi$ from $L$ to $R$ can be extended to an endomorphism of $R$. If $L$ is a direct summand of $R$ then that can be done trivially. If $L$ is not a direct summand then it has a complement $K$ and $L \oplus K$ is essential and proper in $R$. Clearly $\phi$ can be extended to a homomorphism from $L \oplus K$ to $R$. Hence we can assume that $L$ is essential in $R$. As $L$ is quasi-injective it is invariant under endomorphisms of its injective hull and so it is closed under right multiplication by elements of $R$. If $R e_{i} \cap L$ is denoted by $L_{i}$ then $L=L_{1} \oplus L_{2} \oplus L_{3}$ and

$$
\phi=\left(\begin{array}{lll}
\phi_{11} & \phi_{12} & \phi_{13} \\
\phi_{21} & \phi_{22} & \phi_{23} \\
\phi_{31} & \phi_{32} & \phi_{33}
\end{array}\right)
$$

where $\phi_{i j}: L_{i} \rightarrow R e_{j}$ is the appropriate restriction of $\phi$. Clearly $\phi$ can be extended to be an endomorphism of $R$ provided each $\phi_{i j}$ can be extended to a homomorphism $R e_{i} \rightarrow R e_{j}$. This can clearly be done as $R e_{i} \oplus R e_{j}$ is quasi-injective, being a proper ideal. Hence $R$ is injective and therefore is a $Q$-ring.

THEOREM 2.3. A non-local ring is a $p Q$-ring if, and only if, it is either a $Q$-ring or the ring $\left(\begin{array}{ll}D & 0 \\ D & D\end{array}\right)$, for some skew field $D$.

Proof: By Lemmas 2.1 and 2.2 it may be assumed that $R$ has exactly two orthogonal primitive idempotents $e_{1}$ and $e_{2}$. If $R$ decomposes then it is a product of two $Q$-rings and so is itself a $Q$-ring. Hence it may be assumed that $R$ is indecomposable. If $R e_{1} \cong R e_{2}$ then, since $R e_{1}$ is quasi-injective, $R$ is quasi-injective and hence is a $Q$ ring. So it will be assumed that $R e_{1} \not R e_{2}$. Since $R$ is indecomposable one of $e_{1} R e_{2}$, 
$e_{2} R e_{1}$ is non-zero, say $e_{2} R e_{1} \neq 0$. If $L$ is an essential proper submodule of $R e_{1}$ then $L \oplus R e_{2}$ is quasi-injective, hence invariant under all endomorphisms of $E\left(L \oplus R e_{2}\right) \supseteq R$ [2, Proposition 3.1] and thus $L \oplus R e_{2}$ is a right ideal of $R$. The proof of Lemma 2 of [3] now shows that $e_{2} R e_{1} \subseteq S\left(R e_{1}\right) \neq 0$.

In fact, $S\left(R e_{1}\right)$ is simple and essential in $R e_{1}$. To prove this it is sufficient to show that an indecomposable quasi-injective module $M$ must be uniform. If it is not then the map which kills the complement of a non-essential submodule $K$ of $M$ and is the identity on $K$ can be extended to an endomorphism of $M$ which, by Theorem 5.1 and Proposition 5.8 of [2], must be an automorphism - a contradiction. Therefore $S\left(\operatorname{Re}_{1}\right)$ is simple and essential in $R e_{1}$.

By the second paragraph of the proof of Lemma 4 of [3], this minimal submodule is the unique proper submodule of $R e_{1}$ and is the set $e_{2} R e_{1}$. As $R e_{1}$ is quasi-injective $e_{2} R e_{1}$ is a one dimensional right vector space over $e_{1} R e_{1}$ and a simple left $e_{2} R e_{2}$ module. If $e_{1} R e_{2}=0$ then by the proof of Lemma 4 of [3], $e_{2} R e_{2}=R e_{2}$ is a skew field and $R \cong\left(\begin{array}{cc}D & 0 \\ D & D\end{array}\right)$ where $D \cong e_{2} R e_{2} \cong e_{1} R e_{1}$. If $e_{1} R e_{2} \neq 0$ then, as for $R e_{1}$, it follows that $R e_{2}$ has a unique submodule which is the set $e_{1} R e_{2}$ and is a one dimensional right vector space over the skew field $e_{2} R e_{2}$. It can now be shown, as in [3], that $R \cong\left(\begin{array}{ll}D & V \\ V & D\end{array}\right)$ where $D \cong e_{1} R e_{1} \cong e_{2} R e_{2}$ is a skew field and $V$ is a null $D$-algebra, one dimensional on both sides. Thus $R$ is a $Q$-ring, by Theorem 3 of [3]. This proves the theorem.

We now turn to $w Q$-rings.

If a $w Q$-ring decomposes (as a ring) then it is a $Q$-ring as each summand is quasiinjective and thus a $Q$-ring. Therefore it is only necessary to consider indecomposable $w Q$-rings. Since principal ideal domains are trivially $w Q$-rings (that is, by default) the main interest is in $w Q$-rings with more than one idempotent.

THEOREM 2.4. If $R$ is a $w Q$-ring with finite identity and more than one idempotent then $R$ is a $p Q$-ring.

Proof: Let $1=e_{1}+\cdots+e_{n}, n \geqslant 2$, be a decomposition of the identity of $R$ into orthogonal primitive idempotents. We want to show that every proper left ideal is quasi-injective. Clearly each $R e_{i}$ is quasi-injective so if all the $R e_{i}$ are isomorphic $R$ is itself quasi-injective and is therefore a $Q$-ring. So we may assume that all the $R e_{i}$ are not mutually isomorphic. Assume that $e_{1} R e_{2} \neq 0$ and let $K=R a$ for some non-zero $a \in e_{1} R e_{2}$. If $K \oplus R e_{1} \cong R$ then $n=2$ and $K \cong R e_{2}$. It follows that $R e_{2} \cong R e_{1}$ since by the projectivity of $R e_{2}$, the isomorphism from $R e_{2}$ to $K$ can be factored through $R e_{1} \rightarrow K$. But this case has been excluded by assumption. Therefore $K \oplus R e_{1}$ is quasi-injective. By Proposition 3.1 of [2], $K \oplus R e_{1}$ is invariant under endomorphisms 
of its injective hull and so is invariant under endomorphisms of $R e_{1} \oplus R e_{2}$. That is, $K \oplus R e_{1}$ is closed under right multiplication by elements of $\left(e_{1}+e_{2}\right) R\left(e_{1}+e_{2}\right)$. Therefore $e_{1} R e_{2}=e_{1}^{2} R e_{2} \subseteq K$. As $R e_{2}$ is indecomposable and quasi-injective it is uniform, so $K$ is simple. Consequently, if $i \neq j \neq k$ then $e_{i} R e_{j} \cdot e_{j} R e_{k}=0$ and $e_{i} R e_{j} \cdot e_{j} R e_{i}=0$.

Let $\phi: R \rightarrow L$ be an isomorphism to a left ideal $L$ of $R$. Then for each $i$, $e_{i} \phi=a_{i} e_{i}+\sum_{j \neq i} b_{i j} e_{j}$ for some $a_{i} \in e_{i} R e_{i}, b_{i j} \in e_{i} R e_{j}$. Since each $b_{i j}$ generates a simple or zero left ideal, $a_{i} \neq 0$. As $e_{i} R e_{i}$ is a local ring, $a_{i}$ must be a unit in $e_{i} R e_{i}$ [2, Proposition 5.8]. If $a_{i}^{-1}$ is the inverse of $a_{i}$ in $e_{i} R e_{i}$, then $g_{1}=b_{1 i} a_{i}^{-1} e_{i} \phi=$ $b_{1 i} e_{i}+\sum_{j \neq i} b_{1 i} a_{i}^{-1} b_{i j} e_{j}=b_{1 i} e_{i}$, since $b_{1 i} a_{i}^{-1} b_{i j} \in e_{1} R e_{i} . e_{i} R e_{j}, i \neq j$. Therefore $e_{1} \phi-$ $g_{1}-\cdots-g_{n}=a_{1} e_{1} \in L$. This means that $R e_{1} \subseteq L$. Similarly it can be shown that each $R e_{i} \subseteq L$ and therefore $R=L$. That shows that $R$ has no proper left ideals isomorphic to itself and therefore all proper left ideals are quasi-injective. That is, $R$ is a $p Q$-ring.

\section{REFERENCES}

[1] K. A. Byrd, 'Right self-injective rings whose essential right ideals are two-sided', Pacific J. Math 82 (1979), 23-41.

[2] C. Faith, Lectures on injective modules and quotient rings, Lecture Notes in Mathematics 49 (Springer-Verlag, Berlin, Heidelberg, New York, 1967).

[3] G. Ivanov, 'Non-local rings whose ideals are all quasi-injective', Bull. Austral. Math. Soc. 6 (1972), 45-52.

[4] G. Ivanov, 'Non-local rings whose ideals are all quasi-injective: addendum', Bull. Austral. Math. Soc. 12 (1975), 159-160.

[5] S. Mohamed and S. Singh, 'Rings in which proper right ideals are quasi-injective', preprint, Amer. Math. Soc. Notices 22 (1975), A621.

[6] S. Mohamed and S. Singh, 'Weak q-rings', Canad. J. Math 24 (1977), 687-695.

[7] S. Mohamed and S. Singh, 'Weak q-rings with zero singular ideal', Proc. Amer. Math. Soc. 76 (1979), 25-30.

School of Mathematics, Physics,

Computing and Electronics

Macquarie University

New South Wales 2109

Australia 